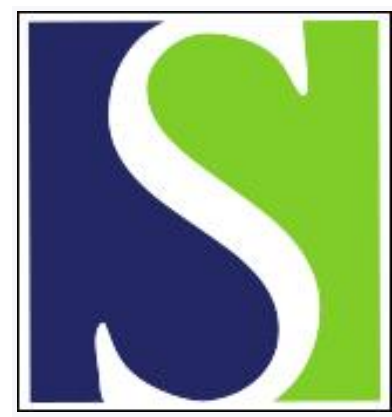

Scand J Work Environ Health 2017;43(6):578-586

https://doi.org/10.5271/sjweh.3668

Published online: 15 Sep 2017, Issue date: 01 Nov 2017

Night work, long work weeks, and risk of accidental injuries. A register-based study

by Larsen AD, Hannerz H, Møller SV, Dyreborg J, Bonde JP, Hansen J, Kolstad HA, Hansen ÅM, Garde AH

Danish employees with night work had a higher ratio of accidental injuries compared to day workers. Awareness should be raised in order to prevent injuries in the future. No association between weekly working hours and accidental injuries were found. Thus it appears that the current regulation ensures that long weekly working hours does not imply an increased risk of injuries.

Affiliation: The National Research Centre for the Working Environment, Lersoe Parkalle 105, DK-2100 Copenhagen. adl@nrcwe.dk

Refers to the following texts of the Journal: 2003;29(3):171-188 2005;31(5):329-335 2006;32(3):232-240 2011;37(3):173-185 2015;41(3):268-279

The following articles refer to this text: 2020;46(5):480-487; 2021;47(3):191-199; 2021;47(6):415-424

This article in PubMed: www.ncbi.nlm.nih.gov/pubmed/28914325 


\title{
Night work, long work weeks, and risk of accidental injuries. A register- based study
}

\author{
by Ann D Larsen, PhD, ${ }^{1}$ Harald Hannerz, PhD, ${ }^{1}$ Simone V Møller, MSc, ${ }^{1}$ Johnny Dyreborg, PhD, ${ }^{1}$ \\ Jens Peter Bonde, MD, ${ }^{2}$ Johnni Hansen, PhD, ${ }^{3}$ Henrik A Kolstad, PhD, ${ }^{4}$ Åse Marie Hansen, PhD, ${ }^{1,5}$ \\ Anne Helene Garde, PhD ${ }^{1,5}$
}

\begin{abstract}
Larsen AD, Hannerz H, Møller SV, Dyreborg J, Bonde JP, Hansen J, Kolstad HA, Hansen ÅM, Garde AH. Night work, long work weeks, and risk of accidental injuries. A register-based study. Scand J Work Environ Health. 2017;43(6):578-586. doi:10.5271/sjweh.3668

Objectives The aims of this study were to (i) investigate the association between night work or long work weeks and the risk of accidental injuries and (ii) test if the association is affected by age, sex or socioeconomic status.

Methods The study population was drawn from the Danish version of the European Labour Force Survey from 1999-2013. The current study was based on 150438 participants (53\% men and 47\% women). Data on accidental injuries were obtained at individual level from national health registers. We included all 20-59-year-old employees working $\geq 32$ hours a week at the time of the interview. We used Poisson regression to estimate the relative rates (RR) of accidental injuries as a function of night work or long work weeks ( $>40$ hours per week) adjusted for year of interview, sex, age, socioeconomic status (SES), industry, and weekly working hours or night work. Age, sex and SES were included as two-way interactions.
\end{abstract}

Results We observed 23495 cases of accidental injuries based on 273700 person years at risk. Exposure to night work was statistically significantly associated with accidental injuries (RR 1.11, 99\% CI 1.06-1.17) compared to participants with no recent night work. No associations were found between long work weeks $(>40$ hours) and accidental injuries.

Conclusion We found a modest increased risk of accidental injuries when reporting night work. No associations between long work weeks and risk of accidental injuries were observed. Age, sex and SES showed no trends when included as two-way interactions.

Key terms accident; Denmark; Labour Force Survey; prospective study; shift work; working time arrangement.

Living in a society with the demands of 24-hour availability implies that employees in, eg, service, transportation, security, and healthcare sectors have working time arrangements outside conventional daytime hours, including shift and night work, and long work weeks. In 2015 , about $19 \%$ of all European employees worked nights at least once a month, approximately $23 \%$ reported working in shifts and about $25 \%$ worked $>40$ hours per week (1).

Work at night has been associated with higher risk of injuries compared to daytime work, eg, among nurses
(2), offshore workers (3) and construction workers (4). Several studies have shown that long shift duration is associated with increased risk of accidents (4-6), but in contrast only few studies have assessed the effects of long work weeks (7). The literature of working time arrangements and risk of accidental injuries is dominated by cross-sectional studies, where exposure to working hour arrangement and injuries have been assessed at the same time rendering the design retrospective and leaving the risk of reporting bias. Only a few longitudinal studies have included shift work either as a

1 The National Research Centre for the Working Environment, Copenhagen, Denmark.

2 Department of Occupational and Environmental Medicine, Bispebjerg-Frederiksberg University Hospital, Copenhagen, Denmark.

3 Danish Cancer Society Research Center, Copenhagen, Denmark.

4 Department of Occupational Medicine, Danish Ramazzini Centre, University of Aarhus, Aarhus, Denmark.

5 Department of Public Health, University of Copenhagen, Copenhagen, Denmark.

Correspondence to: Dr. A.D. Larsen, The National Research Centre for the Working Environment, Lersoe Parkalle 105, DK-2100 Copenhagen. [Email: adl@nrcwe.dk] 
single exposure (4) or divided into evening, rotating, or night shifts (8). The two latter studies found a positive association to self-reported accidental work injuries (4, 8). Accidental injuries have mainly been assessed by self-report, whereas register data on work accidents has been used only in a few studies. A cross-sectional study by Rodrigues et al (9) included 51 male offshore workers reporting higher relative risk of accidents during late evening and night and a register study of Lauridsen et al (10) showed no difference between day and night shifts in terms of injury rates but an increase in number of accidental injuries between 00:00-06:00 compared to 18:00-24:00 hours (10). Tüchsen et al has studied risk of accidents in relation to commuting by investigating the role of living in building-site camps versus daily commuting from home to construction sites. The study found that those living in building-site camps suffered fewer accidental injuries during work than those commuting daily (11).

There are more than 3.2 million non-fatal work accidents with at least four calendar days of absence from work and 3739 fatal work accidents in the European countries (12). There is therefore still a need for knowledge on causes of accidents and thus large cohort register studies of associations between shift work and long working hours, on the one hand, and accidents, on the other, are warranted.

A mechanism behind working hour arrangements and the risk of accidental injuries is suggested to go through sleepiness or fatigue [reviewed in (13)], which are well-known consequences of shift and night work, as well as long work weeks [reviewed in (14-16)]. A key element is sleep, which is determined by two independent biological mechanisms: the circadian rhythm or "body clock", where sleepiness peaks in the early morning, usually between 02:00-06:00 hours, and "sleep pressure", which expresses the amount of fatigue that gradually accumulates during the course of being awake, due to mental and physical activity (17). Thus sleepiness and fatigue can be affected both by disturbing the circadian rhythm, as the case with night work, and by extended physical and mental activities due to long working hours. When persons are affected by sleepiness or fatigue, their attention and decision-making abilities during work activities, on the road or in relation to home or leisure-time activities, can become seriously weakened. However, the evidence of a causal pathway from shift work through sleepiness to accidental injuries remains unestablished due to limited epidemiological support [reviewed in (18)].

So far the main focus has been on work-related accidents, but accidents occur both during and outside work eg, during commuting and at home during leisure time. Night work and long working hours affect alertness and cognitive efficiency both acutely during working hours and the following days (19-23). Thus, working hours is expected to affect not only the risk of work-related accidents, but also risk of accidents after work eg, during commuting particularly from work as well as during leisure time.

We therefore aim to investigate the association of night work and long work weeks on the risk of accidental injuries causing hospital contact or death during work, commuting or home, and leisure activities in combination. Further, we aim to test if the association between night work and long work weeks and accidental injuries is affected by age, sex or socioeconomic status (SES). All analyses will be based on a randomly selected sample, which to our knowledge is the largest study sample ever used to analyze associations between working time arrangements and risk of accidental injuries.

\section{Methods}

\section{Data}

The study is protocol-based hence an elaborated description of data and methods can be found here (24).We used data from the Danish Labour Force Survey (DLFS) from 1999-2013 linked with register data on hospital contact or death due to accidental injuries. The survey data collection was carried out by Statistics Denmark conducting interviews with a random sample of Danish citizens 15-74 years of age as part of the European Labour Force Surveys (ELFS). The samples are drawn four times per year and the same participant is invited to be interviewed four times over a period of one and a half year (25). It should be noted that our database does not contain any information on what time of the day the accident occurred nor whether or not it occurred at work.

The Danish Data Protection Agency (j.no 2015-570074) approved the study.

\section{Selection of study participants}

There were a total of 357085 participants participating in the DLFS in the years 1999-2013. We excluded participants aged $<20$ and $>59$ years at the start of their respective follow-up period (early retirement at age 60 years was a possibility in the mentioned time period). We excluded unemployed and retired participants as they have no exposure of interest in this study. We also excluded participants working either $<32$ hours (part-time jobs could be due to health problems) or $>100$ hours per week (outliers). Further, we excluded participants with missing data on exposure from the survey or outcome from the registers. In total, 150438 participants were included in the analyses. The flow is given in figure 1. 


\section{Working time arrangements}

Questions regarding number of weekly working hours and night work are included in the DLFS. In the primary analysis, the participants' working time arrangement was classified in accordance with the responses given in their first interview if interviewed more than once. The calculated weekly working hours were based on participants' usual working hours and included secondary jobs. The variable was treated as a categorical variable with: 32-40 hours representing normal weekly working hours, which served as the reference, 41-48 hours representing overtime within the limits of the European Working Time Directive and 49-100 hours representing overtime work beyond the threshold of the directive.

Exposure to night work was based on the response to the questions: "Do you work at night?" (1994-2000) or "Have you worked night during the last four weeks?" (2001-2013). Positive responses: "yes, regularly (ie, more than half of the workdays in the past 4 weeks)" or "yes, occasionally (ie, at least once during the past 4 weeks, but less than half of the workdays)" were regarded as exposure to night work. If the response was negative ("No, not within the past 4 weeks"), the participants were regarded as unexposed and this served as the reference. The responses "yes, regularly" and "yes, occasionally" were combined to increase power. A post-hoc analysis including the categories separately is included.

There have been a few changes in data collection routines over the years, but these will be disregarded [for more information see protocol (24)].

\section{Accidental injuries causing hospital contact or death}

Based on the participants' unique personal identification numbers, which has been applied to all residents in Denmark since 1968 (26), outcomes were retrieved from the National Patient Registry (NPR) and the Danish National Register of Cause of Death (NRCD).

The NPR contains data on all inpatients from all Danish hospitals since 1977 and, since 1995, also covers outpatients and emergency ward visits (27); since 1994, all diagnoses have been coded according to the ICD-10 (28). The endpoints used for analyses in this study were defined as hospital contacts with a primary diagnosis code in the interval S00-T98 (injury, poisoning and certain other consequences of external causes), excluding the diagnoses T80-T88 (complications of surgical and medical care, not elsewhere classified). A detailed list of the diagnostic subgroups that are included in the case definition is given in our study protocol (24). The NRCD contains information on deceased persons with residence in Denmark, including manner and cause of death (29). For this study, primary diagnosis in the interval V01-Y98 (external causes of morbidity and

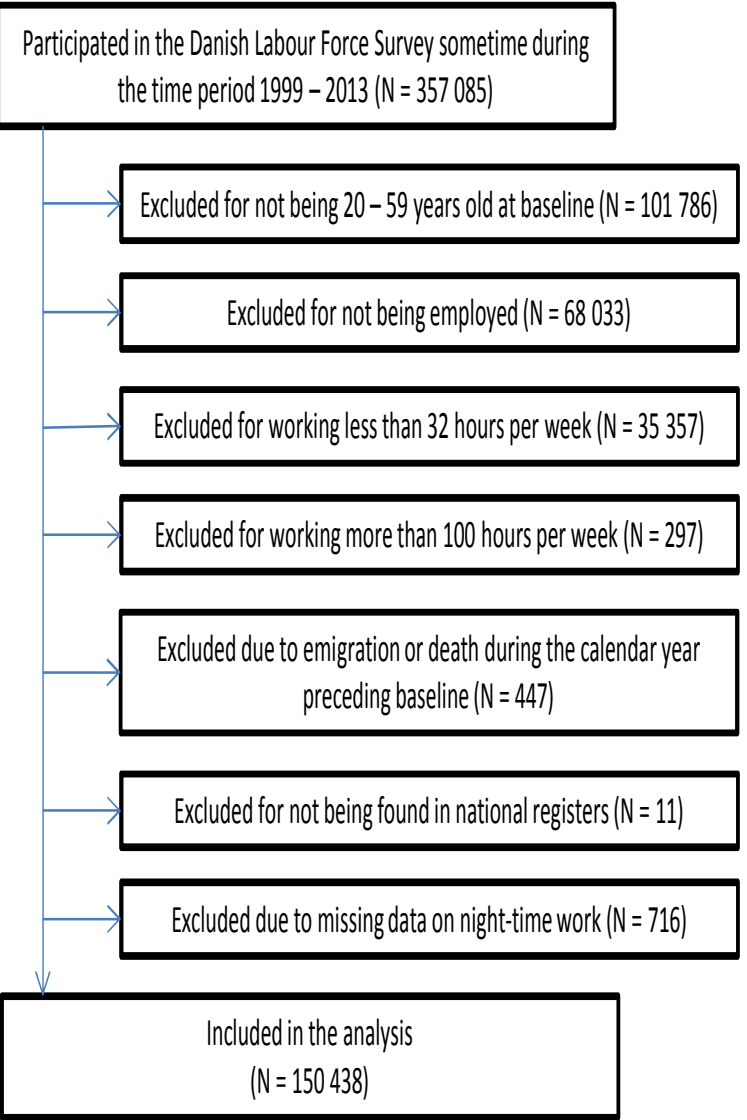

Figure 1. Flowchart of study participants.

mortality) were included and Y40-Y84 (complications of surgical and medical care) and X60-X84 (intentional self-harm) were excluded.

The two outcome categories were combined as we were not interested in severity but wanted to include all cases of injuries, fatal as well as non-fatal. Since there were only 30 fatal accidental injuries retrieved from the NRCD, and 23465 non-fatal accidental injuries retrieved from the NPR, we cannot test for fatal accidental injuries only due to lack of power.

\section{Measurement of potential confounders}

From the DLFS, we included year of interview (19992000; 2001-2006; 2007-2013). Information on sex, age at the start of their respective follow-up period (20-24; $25-29 ; 30-39 ; 40-49 ; 50-59$ years), death, and migration were included from the Civil Registration System (30).

Information on SES, occupation, and industry were included from the Employment Classification Module, which Statistics Denmark has maintained since 1975 and updates every year (31), SES and industry refers to the participants' main activity during the year of the inter- 
view. In accordance with the three-class version of the European Socioeconomic Classification (ESeC), SES was divided into high (managers), medium (intermediate), and low (working class) (32). A detailed description of the coding procedure is given elsewhere (33).

Industries were coded in accordance with the three classifications covering the three periods: DB93 in 1999-2002 (34), DB03 in 2002-2007 (35), and DB07 in the calendar years 2008-2013 (36). The industrial groups covered (i) agriculture and fishing, (ii) manufacturing, (iii) construction, (iv) wholesale and retail trade, (v) transport and storage, (vi) accommodation and food service, (vii) human health and social work, (viii) other, and (ix) missing.

\section{Statistical models and tests}

We used Poisson regression to estimate the relative rates (RR) of accidental injuries as a function of weekly working hours or night work.

The follow-up period for participants who were interviewed in calendar year 1999-2012 ran 24 months from beginning of the following calendar year. The follow-up period for participants who were interviewed in 2013 ran 12 months from the beginning of 2014. The follow-up ended when the participant experienced an accidental injury causing hospital contact or death, emigrated, died of other reasons or by the end of the follow-up period.

Two sets of models tested the effects of (i) night work and (ii) weekly working hours as exposure on the risk of hospital contact or death due to accidental injuries. The main model (model 1) was adjusted for sex, age, age $\times$ sex, year of interview, SES, industry and mutual adjustment for night work or weekly working hours. The additional models included a two-way interaction to the main model: model $2=$ model $1+$ exposure $\times$ age, model $3=$ model $1+$ exposure $\times$ sex, model $4=$ model $1+$ exposure $\times$ SES.

\section{Post-hoc sensitivity analysis - only stable exposure to night-time work}

In order to find out whether the strength of the association between night-time work and risk of injuries increases when exposure is more stable over time, we conducted a post-hoc sensitivity analysis, in which we only included people who (i) participated in more than one interview, (ii) were aged 20-59 years during their last interview, (iii) were employees according to their first as well as their last interview, and (iv) belonged to the same category in relation to night-time work (yes versus no) during their last interview as they did during their first interview. The average lag between the first and last interview was 61.8 weeks. The follow-up of the included participants started on 1 January of the calendar year, which succeeded the calendar year of their last interview. In all other respects, the follow-up and statistical models were the same as in the primary analysis.

Post-hoc sensitivity analysis - occasional versus regular night-time work

In the primary analysis, we used a dichotomized exposure variable for night work ("yes" versus "no"). In this sensitivity analysis, we divided night work into three categories ("no"; "yes, occasionally"; "yes, regularly") and estimated the rate ratios for "yes, occasionally" and "yes, regularly" versus "no". The statistical model and inclusion criteria were the same as in the primary analysis.

\section{Significance levels}

As presented, the above four models for each exposure were planned. The overall significance levels for each of the test of main exposures and risk of accidental injuries were therefore set to be $\leq 5 \%$, where the significance levels for each of the individual tests was $1 \%$.

\section{Results}

Table 1 shows the characteristics of the study population in relation to working time arrangements. Of the total population, $12.8 \%$ reported night work, which was more common among men (65.9\%) than women. Night work was most frequent among the 40-49-year-olds (30.4\%) and least among the 20-24-year-olds (6.2\%). The highest proportion of night work was found in the lowest SES group (47.9\%).

With respect to working time, $9.9 \%$ and $6.0 \%$ of the study population worked $41-48$ and $\geq 49$ hours, respectively per week. Long work weeks were more frequent among men (41-48 hours: $61.7 \%,>48$ hours: $77.2 \%$ ) than women. Long work weeks were most frequent among 40-49-year-olds (41-48 hours: $29.8 \%$, >48 hours: $32.0 \%$ ) and least frequent among the youngest members of the study population (41-48 hours: $6.0 \%$, $>48$ hours: $5.3 \%$ ). Weekly working time of $41-48$ hours was most common among those in the lowest SES group (37.3\%), whereas $>48$ hours per week was most frequent among those in the highest SES group (36.8\%).

We found 23495 cases of hospital contact or death due to injuries in 273700 person years at risk. The overall test with regards to night work and accidental injuries showed a statistically significant association $(\mathrm{P}<0.0001)$, but there were no statically significant association to the interaction term between night work 
Table 1. Participant characteristics with regards to age, sex, and socioeconomic status (SES) for night work and weekly working hours.

\begin{tabular}{|c|c|c|c|c|c|c|c|c|c|c|}
\hline & \multicolumn{4}{|c|}{ Night work } & \multicolumn{6}{|c|}{ Weekly working time (hours per week) } \\
\hline & \multicolumn{2}{|c|}{ Yes } & \multicolumn{2}{|c|}{ No } & \multicolumn{2}{|c|}{$32-40$} & \multicolumn{2}{|c|}{$41-48$} & \multicolumn{2}{|c|}{$>48$} \\
\hline & $\mathrm{N}$ & $\%$ & $\mathrm{~N}$ & $\%$ & $\mathrm{~N}$ & $\%$ & $\mathrm{~N}$ & $\%$ & $\mathrm{~N}$ & $\%$ \\
\hline Total $(\mathrm{N}=150438)$ & 19212 & 12.8 & 131226 & 87.2 & 126619 & 84.2 & 14852 & 9.9 & 8967 & 6.0 \\
\hline \multicolumn{11}{|l|}{ Age (years) } \\
\hline $20-24$ & 1193 & 6.2 & 9224 & 7.0 & 9048 & 7.1 & 890 & 6.0 & 479 & 5.3 \\
\hline $25-29$ & 1939 & 10.1 & 12568 & 9.6 & 12236 & 9.7 & 1495 & 10.1 & 776 & 8.7 \\
\hline $30-39$ & 5605 & 29.2 & 34737 & 26.5 & 33885 & 26.8 & 4146 & 27.9 & 2311 & 25.8 \\
\hline $40-49$ & 5838 & 30.4 & 37792 & 28.8 & 36332 & 28.7 & 4432 & 29.8 & 2866 & 32.0 \\
\hline $50-59$ & 4637 & 24.1 & 36905 & 28.1 & 35118 & 27.7 & 3889 & 26.2 & 2535 & 28.3 \\
\hline \multicolumn{11}{|l|}{ Sex } \\
\hline Men & 12654 & 65.9 & 67330 & 51.3 & 63898 & 50.5 & 9164 & 61.7 & 6922 & 77.2 \\
\hline Women & 6558 & 34.1 & 63896 & 48.7 & 62721 & 49.5 & 5688 & 38.3 & 2045 & 22.8 \\
\hline \multicolumn{11}{|l|}{ SES } \\
\hline High & 5854 & 30.5 & 38178 & 29.1 & 35389 & 27.9 & 5340 & 36.0 & 3303 & 36.8 \\
\hline Medium & 1522 & 7.9 & 26439 & 20.1 & 24449 & 19.3 & 2316 & 15.6 & 1196 & 13.3 \\
\hline Low & 9201 & 47.9 & 53203 & 40.5 & 54061 & 42.7 & 5547 & 37.3 & 2796 & 31.2 \\
\hline Unknown & 2635 & 13.7 & 13406 & 10.2 & 12720 & 10.0 & 1649 & 11.1 & 1672 & 18.6 \\
\hline
\end{tabular}

and age $(\mathrm{P}=0.19)$, sex $(\mathrm{P}=0.09)$, or $\operatorname{SES}(\mathrm{P}=0.15)$. No association of weekly working hours and accidental injuries were observed for neither main effects $(\mathrm{P}=0.09)$ nor in interaction with age $(\mathrm{P}=0.31)$, sex $(\mathrm{P}=0.57)$ or $\mathrm{SES}(\mathrm{P}=0.08)$.

Table 2 presents the results of the main effects of weekly working hours and night work on the risk of hospital contact or death due to injuries. As indicated by the overall P-values for long work weeks, no statistically significant results were found for this exposure. We performed a sensitivity analysis in which we estimated the rate ratio for injury first among workers with 49-60 versus $32-40$ and then among $\geq 49$ versus $32-40$ weekly working hours. At two decimal places, both of these rate ratios equaled the one that was obtained for the contrast 49-100 versus 32-40 hours in our primary analysis. However, exposure to night work was statistically significantly associated with hospital contact or death due to injuries with a rate ratio of 1.11 (99\% CI 1.06-1.17) compared to participants with no night work. In response to a reviewer comment, we used data from table 2 to estimate the excess fraction (37) of night work on the risk of accidental injuries among full-time employees in Denmark at 0.0136 (95\% CI 0.0070-0.0203). There are approximately 2 million full-time employees in Denmark. The data from table 2, thereby imply that approximately 2300 (95\% CI 1200-3500) hospital treated injuries per year may be attributed to night work among full-time employees in Denmark.

Results from the analysis of accidental injuries as a function of night work showed no clear trend of age when stratifying as seen in table 3 . Highest rate ratio was observed among the 25-29-year-olds with night work (RR 1.19, 99\% CI 1.04-1.36) followed by the 40-49 year olds with night work (RR 1.16, 99\% CI 1.06-1.27) compared to the participants in the respective groups without night work. Results from the analysis stratified by sex showed that men working night shift had a rate ratio of 1.09 (99\% CI 1.02-1.15) compared to men without night work, see table 4. Similarly; women with night work had higher rate ratio of accidental injuries (RR $1.17,99 \%$ CI 1.07-1.27) compared to women without night work. Analysis on accidental injuries as a function of night work stratified by SES showed elevated and statistically significant rate ratios with highest rates among those with a medium socioeconomic status (RR 1.22 , 99\% CI 1.03-1.45) (table 5).

Results from the analyses on long work weeks and risk of accidental injuries showed no statistically significant associations when stratifying by age (table 6). Similarly, no statistically significant associations were found when stratifying by sex and SES (results not shown).

Results from the post-hoc sensitivity tests showed that in a sub-population among persons interviewed more than once with stable exposure of night work (7685 persons) or no night work (89 826 persons), night work was associated with accidental injuries (RR 1.14, 99\% CI 1.06-1.24).

When dividing night work into regularly (9391 persons) and occasionally (9821 persons) versus no night work (131 226 persons), the rate ratio was highest among those having regularly night work (RR 1.13, 99\% CI 1.05-1.20), followed by those having night work occasionally (RR 1.09, 99\% CI 1.02-1.17).

\section{Discussion}

The main finding of this study was the association between night work and increased rates of accidental injuries. In contrast, no associations between long work 
Table 2. Rate ratio (RR) with $99 \%$ confidence interval $(\mathrm{CI})$ of accidental injuries among Danish employees 2000-2014-2015, as a function of weekly working hours and night work, respectively

\begin{tabular}{lrrrrr}
\hline Exposure & \multicolumn{5}{c}{$\begin{array}{c}\text { Hospital contact or death due to } \\
\text { accidental injuries }\end{array}$} \\
\cline { 2 - 6 } & Level & $\begin{array}{c}\text { Person years } \\
\text { at risk }\end{array}$ & Cases & RR & $99 \% \mathrm{Cl}$ \\
Weekly working & $>48$ & 16175 & 1484 & 1.02 & $0.95-1.09$ \\
hours $^{\mathrm{a}}$ & $41-48$ & 27062 & 2281 & 0.96 & $0.90-1.01$ \\
& $32-40$ & 230463 & 19730 & 1.00 &. \\
Night work $^{\mathrm{b}}$ & Yes & 34318 & 3451 & 1.11 & $1.06-1.17$ \\
& No & 239383 & 20044 & 1.00 &.. \\
\hline
\end{tabular}

a Adjusted for year of interview, sex, age, night work, socio-economic status and industry.

${ }^{\mathrm{b}}$ Adjusted for year of interview, sex, age, weekly working hours, socioeconomic status and industry.

Table 3. Rate ratio (RR) with $99 \%$ confidence interval $(\mathrm{Cl})$ for accidental injuries as a function of night work among Danish employees 2000-2014-2015, stratified by baseline age group.

\begin{tabular}{lrrrrr}
\hline Baseline & Night & \multicolumn{4}{c}{ Accidental injuries } \\
\cline { 3 - 6 } $\begin{array}{l}\text { age group } \\
\text { (years) }\end{array}$ & work & $\begin{array}{c}\text { Person years } \\
\text { at risk }\end{array}$ & Cases & RR a & $99 \% \mathrm{Cl}$ \\
\hline $20-24$ & Yes & 1958 & 333 & 1.03 & $0.88-1.19$ \\
& No & 15474 & 2440 & 1.00 &.. \\
$25-29$ & Yes & 3295 & 458 & 1.19 & $1.04-1.36$ \\
& No & 22234 & 2388 & 1.00 &.. \\
$30-39$ & Yes & 9958 & 1050 & 1.10 & $1.00-1.20$ \\
& No & 63181 & 5478 & 1.00 &.. \\
$40-49$ & Yes & 10596 & 972 & 1.16 & $1.06-1.27$ \\
& No & 69904 & 5087 & 1.00 &.. \\
$50-59$ & Yes & 8510 & 638 & 1.06 & $0.95-1.18$ \\
& No & 68589 & 4651 & 1.00 &.. \\
\hline
\end{tabular}

a Adjusted for year of interview, sex, weekly working hours, socioeconomic status and industry.

Table 4. Rate ratio (RR) with $99 \%$ confidence interval (CI) for accidental injuries among Danish employees 2000-2014 as a function of night work, stratified by sex.

\begin{tabular}{lrrrrr}
\hline Sex & $\begin{array}{l}\text { Night } \\
\text { work }\end{array}$ & \multicolumn{4}{c}{ Accidental injuries } \\
\cline { 3 - 6 } & & $\begin{array}{c}\text { Person years } \\
\text { at risk }\end{array}$ & Cases & $\mathrm{RR}^{\text {a }}$ & $99 \% \mathrm{Cl}$ \\
\hline \multirow{2}{*}{ Wen } & Yes & 22349 & 2467 & 1.09 & $1.02-1.15$ \\
& No & 120675 & 12037 & 1.00 &.. \\
& Yes & 11968 & 984 & 1.17 & $1.07-1.27$ \\
& No & 118708 & 8007 & 1.00 &.. \\
\hline
\end{tabular}

${ }^{a}$ Adjusted for year of interview, age, weekly working hours, socio-economic status and industry.

weeks and risk of accidental injuries were observed. Age, sex, and SES showed no trends when included as two-way interactions for either exposures and cannot explain any of the associations seen.

Our findings with regards to night work and accidental injuries are in line with previous findings showing increased risk when working nights $(2,4,8-10)$. A review of studies among nurses with focus on needle stick injuries concluded that "...shift work is associated
Table 5. Rate ratio (RR) with $99 \%$ confidence interval (Cl) for accidental injuries as a function of night-time work among Danish employees 2000-2014-2015, stratified by socioeconomic status (SES).

\begin{tabular}{lrrrrr}
\hline SES & Night & \multicolumn{4}{c}{ Accidental injuries } \\
\cline { 3 - 6 } & work & $\begin{array}{c}\text { Person years } \\
\text { at risk }\end{array}$ & Cases & RR a & $99 \% \mathrm{Cl}$ \\
\hline High & Yes & 10731 & 798 & 1.13 & $1.02-1.25$ \\
\multirow{4}{*}{ Medium } & No & 71163 & 4447 & 1.00 &.. \\
\multirow{4}{*}{ Low } & Yes & 2764 & 246 & 1.22 & $1.03-1.45$ \\
& No & 49184 & 3290 & 1.00 &.. \\
Unknown & Yes & 16135 & 1941 & 1.11 & $1.04-1.19$ \\
& No & 94833 & 10120 & 1.00 &.. \\
& Yes & 4688 & 466 & 1.01 & $0.89-1.16$ \\
& No & 24203 & 2187 & 1.00 &..
\end{tabular}

a Adjusted for year of interview, age, sex, weekly working hours, and industry.

Table 6. Rate ratio (RR) with $99 \%$ confidence interval (Cl) for accidental injuries, as a function of weekly working hours among Danish employees 2000-2014-2015, stratified by baseline age group.

\begin{tabular}{lrrrrr}
\hline Baseline age & Weekly & \multicolumn{5}{c}{ Accidental injuries } \\
\cline { 3 - 6 } group (years) & $\begin{array}{c}\text { working } \\
\text { hours }\end{array}$ & $\begin{array}{c}\text { Person } \\
\text { years at } \\
\text { risk }\end{array}$ & Cases & RR a & $99 \% \mathrm{Cl}$ \\
\hline $20-24$ & $>48$ & 783 & 133 & 1.01 & $0.80-1.27$ \\
& $41-48$ & 1508 & 216 & 0.88 & $0.73-1.05$ \\
& $32-40$ & 15141 & 2424 & 1.00 &.. \\
$25-29$ & $>48$ & 1310 & 186 & 1.08 & $0.88-1.32$ \\
& $41-48$ & 2627 & 299 & 0.97 & $0.83-1.14$ \\
$30-39$ & $32-40$ & 21598 & 2361 & 1.00 &.. \\
& $>48$ & 4126 & 432 & 1.04 & $0.91-1.18$ \\
& $41-48$ & 7530 & 660 & 0.93 & $0.84-1.03$ \\
$40-49$ & $32-40$ & 61483 & 5436 & 1.00 &.. \\
& $>48$ & 5263 & 407 & 0.97 & $0.85-1.11$ \\
$50-59$ & $41-48$ & 8141 & 652 & 1.04 & $0.94-1.16$ \\
& $32-40$ & 67097 & 5000 & 1.00 &.. \\
& $>48$ & 4693 & 326 & 1.04 & $0.89-1.20$ \\
& $41-48$ & 7263 & 454 & 0.91 & $0.80-1.03$ \\
& $32-40$ & 65144 & 4509 & 1.00 &.. \\
\hline
\end{tabular}

a Adjusted for year of interview, sex, night work, socio-economic status and industry.

with a higher incidence or risk of sustaining work related injuries". However, the level of evidence was rather low (2). Based on two studies among offshore workers, Fossum et al's review concluded that shift work, and in particular night work, increased the RR of work accidents (3). In his study, Dong found that construction workers had an elevated risk of accidents when their work schedule included shift work, further, they found a statistically significant association of starting work early (before 07:00 hours) and shift work (4). Dembe et al found elevated hazard ratios (HR) of all nonstandard shift schedules not including split shifts. For night work, they found an increased HR of 1.30 (95\% CI 1.12-1.62) (8). The result from the current study is a bit more modest in the risk estimates compared to the current literature, which to some extent can be explained by the high risk populations of offshore and construction workers 
included in the previous literature.

The study does however, not support previous findings of long work weeks and associations with workrelated injuries (7). There can be several explanations for this. Firstly, Dembe et al's study included only workrelated injuries (7), where we in our study included all injuries covering both motor vehicle crashes, workrelated injuries (if treated at a hospital) as well as leisure time injuries. This has been done as we assume that long work weeks are associated with sleepiness which may increase the risk of accidental injuries also outside work eg, in transportation after work and in leisure time. The results from the studies may therefore not be comparable with current literature.

\section{Strengths and limitations}

Previous studies are mainly based on cross-sectional design and self-reported accidents or injuries. We use a prospective study design and objective register information on accidental injuries, which may be more accurate and less sensitive to recall and diagnostic bias. In contrast to reporting of work injuries, where underreporting has been estimated to be around $50 \%$ (38), registration of hospital contacts is expected to be completed virtually. Another advantage of the study is the large study population that allowed us to study objective outcomes as hospital contact or death due to injuries, although these are much rarer than minor injuries not treated at hospitals.

All analyses were based on a randomly selected sample from the Danish working population and not solely looking at specific industries, which increase the external validity and generalizability.

There are, however, also some limitations which need to be addressed: information on working time is based on self-reports once during the time of the study, which may increase the risk of exposure misclassification. A recent study from Finland showed however, that self-reported working hours compared with objective register-based information on working time in general is non-differential (39). Further the post-hoc sensitivity analysis among workers reporting stable night work exposure when interviewed more than once supports the main findings of an association between night work and increased rates of accidental injuries. Finally, we used a relatively short ( $<2$ years) follow-up time to reduce the risk of participants changing jobs or working hours within the follow-up period. However, this also implies that we are not able to address any questions in regards to short-term effects (within days) of night work and the risk of work accidents.

We assume that workers have more accidental injuries in spare time than at work. However, we were not able to distinguish between accidental injuries at work, on the road, or at home and during leisure time. Further, we assume that the causal pathway from shift work through sleepiness to accidental injuries remains the same for activities related to work, road traffic, or in relation to home or leisure time. However, the impact of this mechanism on the risk of accidents might well be different for work, traffic, and home and leisure-time activities, as the environmental risks are not the same, eg, the work compared to the road traffic environment. Even though we found that night work increases the risk of accidental injuries, the magnitude-of this risk may depend on the hazard sources associated with persons' activities in the three environments, ie, work, traffic, home and leisure-time activities. We have no reason to suspect that this hazard pattern should differ systematically from workers not working at night.

The primary analysis used a dichotomized night work variable. As this may be too crude and cover up any dose-response associations a post-hoc sensitivity analysis included night work both regularly and occasionally showing a tendency of the more night work the higher rates of accidental injuries, although the confidence intervals were overlapping. The findings of a dose-response further strengthen our findings of an association between night work and accidental injuries.

As information on accidental injuries is retrieved from the NPR and the NRCD, we only included injuries severe enough to be diagnosed at a hospital. Minor accidental injuries, both occupational and non-occupational, are therefore not included and this may affect the results. Yet, the mechanisms are expected to be similar and the results from the study of more severe accidents are therefore assumed to be valid also for minor injuries.

Due to lack of power we are unable to investigate non-fatal and fatal accidental injuries separately. As this may be related to a hypothesis on severity, we addressed the issue by adjusting for known factors related to severity as age, sex, SES and industry (40).

We were unable to include all relevant covariates: smoking, alcohol etc, although these have been reported to be associated with work accidents (41-43). Instead, we included SES as a crude proxy for these covariates, as it known that the lower SES, the higher number of non-healthy lifestyle choices. (44).

The present study classified participants as night workers if (in response to a questionnaire in which the term "night" was undefined) they reported to have worked at "night" either regularly or occasionally during the four-week period preceding the interview. In contrast, the EU Working Time Directive (and the Danish implementation hereof) defines "night time" as "any period of at least 7 hours, which includes the period between midnight and 5:00 AM", and it defines "night worker" as "any worker, who, during night-time, work at least three hours of his daily working time as a nor- 
mal course" or "any worker who is likely to work at night-time at least 300 hours during a period of twelve months" (45). It is therefore reasonable to believe that some of the night workers in the present study would not qualify as night workers in a strictly legal sense.

Lastly, the DLFS response rate has decreased with time, from $70 \%$ in 2002 to $53 \%$ in 2013 , which may cause selection bias. It is, however, somewhat improbable that participation in the survey should be associated with the likelihood of experiencing an accidental injury. There is a possibility, however, that participation may depend on exposure to night work or work weeks. There are therefore as with most surveys, a risk of participation bias for which the direction is unknown.

To conclude, we found that participants with night work in a large Danish cohort of employees had a higher rate of accidental injuries compared to participants without night work. Awareness of this should be raised in order to prevent injuries in the future. No association between long works week and accidental injuries were found. Age, sex, and SES showed no trends when included as two-way interactions

To our best knowledge, this is by far the largest prospective cohort study done on working time arrangements and risk of accidental injuries based on a sample that has been randomly selected from a general population.

\section{Acknowledgements}

The authors declare no conflicts of interest.

The Danish Working Environment Research Fund funded this study under grant number 38-201309/20130069288. The Research Fund had played no role in planning, execution or interpretation of the study or in the decision of publishing. The authors thank Otto Melchior Poulsen at the National Research Centre for the Working Environment (NRCWE) for his inspiration and support during initiation of the project. We thank Jesper Møller Pedersen (NRCWE) for assistance with data and contact to Statistics Denmark.

\section{References}

1. Eurofound. Sixth European Working Conditions Survey 2015. Available from: https://www eurofound europa eu/surveys/ data-visualisation/sixth-european-working-conditionssurvey-2015. 2015.

2. Zhao I, Bogossian F, Turner C. Shift work and work related injuries among health care workers: A systematic review. Aust J Adv Nurs. 2010;27(3):62-74.

3. Fossum IN, Bjorvatn B, Waage S, Pallesen S. Effects of shift and night work in the offshore petroleum industry: a systematic review. Ind Health. 2013;51(5):530-44. https://doi. org/10.2486/indhealth.2013-0054.

4. Dong X. Long workhours, work scheduling and work-related injuries among construction workers in the United States. Scand J Work Environ Health. 2005;31(5):329-35. https://doi. org/10.5271/sjweh.915.

5. Barger LK, Cade BE, Ayas NT, Cronin JW, Rosner B, Speizer $\mathrm{FE}$ et al. Extended work shifts and the risk of motor vehicle crashes among interns. N Engl J Med. 2005;352(2):125-34. https://doi.org/10.1056/NEJMoa041401.

6. Wagstaff AS, Sigstad Lie JA. Shift and night work and long working hours--a systematic review of safety implications. Scand J Work Environ Health. 2011;37(3):173-85. https://doi. org/10.5271/sjweh.3146.

7. Dembe AE, Erickson JB, Delbos RG, Banks SM. The impact of overtime and long work hours on occupational injuries and illnesses: new evidence from the United States. Occup Environ Med. 2005;62(9):588-97. https://doi.org/10.1136/ oem.2004.016667.

8. Dembe AE, Erickson JB, Delbos RG, Banks SM. Nonstandard shift schedules and the risk of job-related injuries. Scand J Work Environ Health. 2006;32(3):232-40. https://doi. org/10.5271/sjweh.1004.

9. Rodrigues VF, Fischer FM, Brito MJ. Shift work at a modern offshore drilling rig. J Hum Ergol (Tokyo). 2001;30(1-2):16772 .

10. Lauridsen $\varnothing$, Tønnesen $T$. Injuries related to the aspects of shift working: a comparison of different offshore shift arrangement. J Occup Accid.1990;12:167-76. https://doi. org/10.1016/0376-6349(90)90095-D.

11. Tuchsen F, Hannerz H. Building camps and work related injuries. Occup Environ Med. 2004 April;61(4):370-1. https:// doi.org/10.1136/oem.2002.006783.

12. Eurostat. Accidents at work statistics. Avalable from: http:// ec.europa eu/eurostat/statistics-explained/index php/. Accidents_at_work_statistics 2017.

13. Uehli K, Mehta AJ, Miedinger D, Hug K, Schindler C, Holsboer-Trachsler E et al. Sleep problems and work injuries: a systematic review and meta-analysis. Sleep Med Rev. 2014;18(1):61-73. https://doi.org/10.1016/j. smrv.2013.01.004.

14. Caruso CC. Negative impacts of shiftwork and long work hours. Rehabil Nurs. 2014;39(1):16-25. https://doi. org/10.1002/rnj.107.

15. van der HM. Long workhours and health. Scand J Work Environ Health. 2003;29(3):171-88. https://doi.org/10.5271/ sjweh. 720 .

16. Vedaa O, Harris A, Bjorvatn B, Waage S, Sivertsen B, Tucker $P$ et al. Systematic review of the relationship between quick returns in rotating shift work and health-related outcomes. Ergonomics. 2016;59(1):1-14. https://doi.org/10.1080/0014 0139.2015.1052020.

17. Borbely AA. A two process model of sleep regulation. Hum Neurobiol. 1982;1(3):195-204. 
18. Kecklund G, Axelsson J. Health consequences of shift work and insufficient sleep. BMJ. 2016;355:i5210. https://doi.org/10.1136/bmj.i5210.

19. Ansiau D, Wild P, Niezborala M, Rouch I, Marquie JC. Effects of working conditions and sleep of the previous day on cognitive performance. Appl Ergon. 2008;39(1):99-106. https://doi.org/10.1016/j.apergo.2007.01.004.

20. Folkard S, Tucker P. Shift work, safety and productivity. Occup Med (Lond). 2003;53(2):95-101. https://doi.org/10.1093/ occmed/kqg047.

21. Meijman T, van der MO, van DM. The after-effects of night work on short-term memory performance. Ergonomics. 1993;36(13):37-42. https://doi.org/10.1080/00140139308967852.

22. Smith L, Folkard S, Poole CJ. Increased injuries on night shift. Lancet. 1994;344(8930):1137-9. https://doi.org/10.1016/ S0140-6736(94)90636-X.

23. Tucker P, Folkard S, Macdonald I. Rest breaks and accident risk. Lancet. 2003;361(9358):680. https://doi.org/10.1016/ S0140-6736(03)12566-4.

24. Larsen AD, Hannerz H, Møller S, Dyreborg J, Bonde JP, Hansen J, et al. Study protocol for examining long working hours and night work as risk factors for injuries. Available from: https://figshare.com/s/f7f871f9488535be228a.

25. Statistics Denmark. [Arbejdskraftundersøgelsen]. Available from: www.dst.dk/da/statistik/dokumentation/ statistikdokumentation/arbejdskraftundersoegelsen. 2015.

26. Pedersen CB. The Danish Civil Registration System. Scand J Public Health. 2011;39(7 Suppl):22-5. https://doi. org/10.1177/1403494810387965.

27. Lynge E, Sandegaard JL, Rebolj M. The Danish National Patient Register. Scand J Public Health. 2011;39(7 Suppl):30-3. https://doi.org/10.1177/1403494811401482.

28. World Health Organization (WHO). ICD-10 International Statistical Classification of Diseases and Related Health Problems. 1992.

29. Helweg-Larsen K. The Danish Register of Causes of Death. Scand J Public Health. 2011;39(7 Suppl):26-9. https://doi. org/10.1177/1403494811399958.

30. Pedersen CB. The Danish Civil Registration System. Scand J Public Health. 2011;39(7 Suppl):22-5. https://doi. org/10.1177/1403494810387965.

31. Petersson F, Baadsgaard M, Thygesen LC. Danish registers on personal labour market affiliation. Scand J Public Health. 2011;39(7 Suppl):95-8. https://doi. org/10.1177/1403494811408483.

32. Harrison, E and Rose, D. The European Socio-economic Classification (ESeC) User Guide. Institute for Social and Economic Reaserch, University of Essex, Colchester, UK; 2006.
33. Hannerz H, Larsen AD, Garde AH. Working Time Arrangements as Potential Risk Factors for Ischemic Heart Disease Among Workers in Denmark: A Study Protocol. JMIR Res Protoc. 2016 Apr-Jun; 5(2): e130. https://doi. org/10.2196/resprot.5563.

34. Statistics Denmark. [Dansk Branchekode 1993, 2.udgave]. 1996. Available from: http://www.dst.dk/da/Statistik/ Publikationer/VisPub?cid=4829.

35. Statistics Denmark. [Dansk branchekode 2003 - DB03]. 2002. Available from: http://www.dst.dk/da/Statistik/ Publikationer/VisPub?cid=5132.

36. Statistics Denmark. [Dansk branchekode 2007 - DB07]. 2007. Available from: www.dst.dk/db07.

37. Rothman KJ, Greenland S. Modern Epidemiology. Second edition ed. Philadelphia: Lippincott-Raven Publishers; 1998.

38. Arbejdstilsynet. Anmeldte arbejdsulykker 2009-2014. Copenhagen: Arbejdstilsynet; 2015.

39. Harma M, Ropponen A, Hakola T, Koskinen A, Vanttola P, Puttonen $\mathrm{S}$ et al. Developing register-based measures for assessment of working time patterns for epidemiologic studies. Scand J Work Environ Health. 2015;41(3):268-79. https://doi. org/10.5271/sjweh.3492.

40. Khodabandeh F, Kabir-Mokamelkhah E, Kahani M. Factors associated with the severity of fatal accidents in construction workers. Med J Islam Repub Iran. 2016;30:469.

41. Chau N, Bhattacherjee A, Kunar BM. Relationship between job, lifestyle, age and occupational injuries. Occup Med (Lond). 2009;59(2):114-9. https://doi.org/10.1093/occmed/ kqp002.

42. Dawson DA. Heavy drinking and the risk of occupational injury. Accid Anal Prev. 1994;26(5):655-65. https://doi. org/10.1016/0001-4575(94)90027-2.

43. Laflamme L, Menckel E, Lundholm L. The age-related risk of occupational accidents: the case of Swedish iron-ore miners. Accid Anal Prev. 1996;28(3):349-57. https://doi. org/10.1016/0001-4575(96)00001-2.

44. Social Determinants of Health, 2nd Edition. M Marmot and R Wilkinson (eds). Oxford: Oxford University Press; 2006.

45. DIRECTIVE 2003/88/EC OF THE EUROPEAN PARLIAMENT AND OF THE COUNCIL of 4 November 2003 concerning certain aspects of the organisation of working time.

Received for publication: 2 June 2017 